\title{
Small Logs for Transactional Services: Distinction is much more accurate than (Positive) Discrimination *
}

\author{
Debmalya Biswas, Blaise Genest \\ IRISA/INRIA \& CNRS \\ Campus Universitaire de Beaulieu \\ 35042 Rennes Cedex, France \\ firstname.lastname@irisa.fr
}

\author{
Thomas Gazagnaire ${ }^{1}$ \\ Citrix Systems R\&D Ltd. \\ Black Horse House, Castle Park \\ Cambridge, CB3 OFL, United Kingdom \\ thomas.gazagnaire@citrix.com
}

\begin{abstract}
For complex services, logging is an integral part of many middleware aspects, especially, transactions and monitoring. In the event of a failure, the log allows us to deduce the cause of failure (diagnosis), recover by compensating the logged actions (atomicity), etc. However, for heterogeneous services, logging all the actions is often impracticable due to privacy/security constraints. Also, logging is expensive in terms of both time and space. Thus, we are interested in determining a small number of actions that needs to be logged, to know with certainty the actual sequence of executed actions from any given partial log. We propose two heuristics to determine such a small set of transitions, with services modeled as Finite State Machines. The first one is based on (Positive) Discrimination of transitions, using every observation to know (discriminate) that a maximal number of transitions occurred. We characterize it algebraically, giving a very fast algorithm. The second algorithm, the distinguishing algorithm, uses every observation to maximize the number of transitions which are ensured not to have occurred. We show experimentally that the second algorithm gives much more accurate results than the first one, although it is also slower (but still fast enough).
\end{abstract}

\section{Introduction}

An interesting problem for complex systems is to determine a minimal set of actions that needs to be observable such that a given property holds. Some of the properties studied in literature of discrete event systems are normality [8], observability [7], state observability [10], diagnosability [13], etc. Our system corresponds to a (composite) Web

\footnotetext{
* This work is supported by la Region Bretagne (CREATE ACTIVEDOC) and ANR-06-MDCA-005 DOCFLOW.

${ }^{1}$ Work done while the author was at IRISA, France.
}

service. A Web service [1] refers to an online service accessible via Internet standard protocols. A composite service, composed of already existing (component) services, combines the capabilities of its components to provide a new service. A service schema which specifies the execution order of its components, can be modeled as a Finite State Machine (FSM), performing actions on global variables. We do not tackle here the transformation of a service into a FSM, which should be handled with care to yield a FSM of small size (see [14] and example 1).

Our long-term objective is to provide a transactional framework for (composite) Web services. A transaction [3] can be considered as a group of actions encapsulated by the operations Begin and Commit/Abort, having the following properties: Atomicity (A), Consistency (C), Isolation (I) and Durability (D). Here, we focus on the atomicity aspect, that is, either all the actions of a transaction are executed or none. In the event of a failure, atomicity is preserved by compensation [4]. Compensation consists of executing the compensating actions, corresponding to each executed action of the failed process, in reverse order of the original execution. Thus, for compensation to be feasible, we need to reconstruct each executed action or the complete history of any execution. The usual way of achieving that is to maintain a log of observable actions. However, in addition to the obvious space overhead of logging (in our testing, about 5 times more), the complete log may not always be accessible. For a composite service, the providers of its component services are different. As such, their privacy/security constraints may prevent them from exposing (part of) the logs corresponding to the execution at their sites. Hence, we want from such a partial log to know with certainty the actual sequence of executed actions, to be able to compensate it.

Section 2 introduces the required formal preliminaries including the precise problem statement. Clearly, we are interested in logging the smallest number of transitions possi- 
ble. However, finding [15, 9] or approximating [11] the absolute minimal number of such transitions is NP-Complete. We thus propose our first heuristic in Section 3. The idea behind it is that a logged transition allows to (positively) discriminate several transitions. That is, we optimize the observable set such that every logged transition implies that a maximum number of transitions are sure to have occurred. We then characterize this algorithm in algebraic terms with a matrix, giving a very fast algorithm (see Section 3.2). Our second idea, presented in Section 4, is that a logged transition also allows to distinguish several transitions. That is, we optimize the observable set such that every logged transition implies that a maximum number of transitions did not occur. We test both algorithms experimentally in Section 5. The results show that the distinguishing algorithm gives result from 1 (at least as good, which we prove theoretically) to 10 times smaller than the discriminating algorithm, with an average of 1.9 times smaller.

\section{Preliminaries}

Formally, we model a transactional service as a Finite State Machine (FSM), that is, a 4-tuple $M=$ $\left(Q, s_{0}, s_{f}, \mathcal{T}\right)$, where:

- $Q$ is the finite set of states,

- $s_{0}$ and $s_{f}$ are the initial and final states, respectively,

- $\mathcal{T} \subseteq Q \times Q$ is the (partial) transition relation.

We describe our FSMs as graphs with a unique input and output point, each node and edge corresponds to a state and transition, but we ignore the alphabet. We assume that the service $M$ does not have any unreachable states and that all states can reach the final state $s_{f}$. For a state $q,{ }^{*} q\left(q^{*}\right)$ denotes the set of incoming (outgoing) transitions to (from) $q$. For convenience, we also assume that there are no outgoing transitions from $s_{f}$ and no incoming transitions to $s_{0}$ (Notice that we could deal with a service without these requirements, but the proof would be more technical.) For a transition $\tau=\left(q_{1}, q_{j}\right), q_{i}$ and $q_{j}$ are referred to as the source and target states of $\tau$. We say that an execution sequence $\rho=\tau_{1} \cdots \tau_{n} \in \mathcal{T}^{*}$ is a path of $M$ if there exists $q_{0}, \cdots, q_{n} \in Q^{n+1}$ with $\tau_{i}=\left(q_{i-1}, q_{i}\right)$ for all $1 \leq i \leq n$. A path is called initial if furthermore $q_{0}=s_{0}$. We denote by $\mathcal{P}(M)$ the set of initial paths in $M$. Finally, we denote by $|M|$ the size of $M$, that is, its number of transitions.

Under restricted observability, for a service $M=$ $\left(Q, s_{0}, s_{f}, \mathcal{T}\right)$, only a subset of its transitions $\mathcal{T}_{O} \subseteq \mathcal{T}$ are observable, that is, can be logged. In general, for any execution $\rho$, we call observation projection, the observation we have after $\rho$ was executed (a sequence of transitions, control points, data ..., etc.). We say that an observable projection $\sigma$ is uncertain if there exists two paths having the same projection. The FSM $M$ is execution sequence detectable iff none of its observable projections are uncertain.

Definition 1 For an FSM $M$, let $\mathcal{T}_{O} \subseteq \mathcal{T}$ be the set of observable transitions. The observation projection $\mathrm{Obs}_{O}$ : $\mathcal{T}^{*} \longrightarrow \mathcal{T}_{O}^{*}$ is the morphism with $\operatorname{Obs}_{O}(a)=$ a if $a \in \mathcal{T}_{O}$, and $\operatorname{Obs}_{O}(a)=\epsilon$ if $a \in \mathcal{T} \backslash \mathcal{T}_{O}$, with $\epsilon$ the empty word.

That is, $\operatorname{Obs}_{O}(\rho)$ is the subsequence of $\rho$ obtained by eliminating from $\rho$ every occurrence of a transition which is not in $\mathcal{T}_{O}$. With such an observation projection $\mathrm{Obs}_{O}$, the only way of having execution sequence detectability is to have every transition observable. Indeed, as soon as there exists even one non-observable transition, the service is not execution sequence detectable. Else, let us take a path $\rho \tau$ with the last transition $\tau \notin \mathcal{T}_{O}$. Then, $\operatorname{Obs}_{O}(\rho \tau)=$ $\operatorname{Obs}_{O}(\rho)$. A usual way to overcome such a problem is to ask for certainty only up to the last few events of the sequence [10]. However, this turnaround does not make sense in our framework since if we cannot compensate the very last action, then we cannot compensate any action at all. As such, we design a new observation mechanism, where the last control point reached before failure is monitored, even if the last action is not logged. In practice, it means that every state that is reached is monitored, and overstack the previous state in a special memory buffer.

Definition 2 Let $M$ be an FSM, $\mathcal{T}_{O} \subseteq \mathcal{T}$. The observation projection $\mathrm{Obs}_{O}^{\text {last }}: \mathcal{T}^{*} \longrightarrow\left(\mathcal{T}_{O}^{*}, Q\right)$ is the function $\operatorname{Obs}_{O}^{\text {last }}(\rho)=\left(\operatorname{Obs}_{O}(\rho), q\right)$ for all $\rho \in \mathcal{P}(M)$ ending in $q$.

We will stick with this definition of observability for the rest of the paper. As mentioned before, we are interested in logging as few transitions as possible.

Problem statement. Given an FSM $M=\left(Q, s_{0}, s_{f}, \mathcal{T}\right)$, we call $\mathcal{T}_{O}$ an observable set of transitions if the service is execution sequence detectable with $\mathrm{Obs}_{O}^{\text {last }}$. We want to determine an observable set of transitions $\mathcal{T}_{O} \subseteq \mathcal{T}$ of minimal size. We refer to such a set as a minimal observable set.

The cardinality of a minimal observable set $\mathcal{T}_{O}$ of an FSM $M$ is referred to as its observable size $M O(M)=$ $\left|\mathcal{T}_{O}\right|$. Notice that as is usual with decision and computation algorithms, it is sufficient to have an algorithm which from an FSM gives its observable size. That is, we can derive a minimal observable set of the FSM based on an algorithm answering whether its observable size is bigger than $n$, for any $n$, and in time polynomially equivalent. 


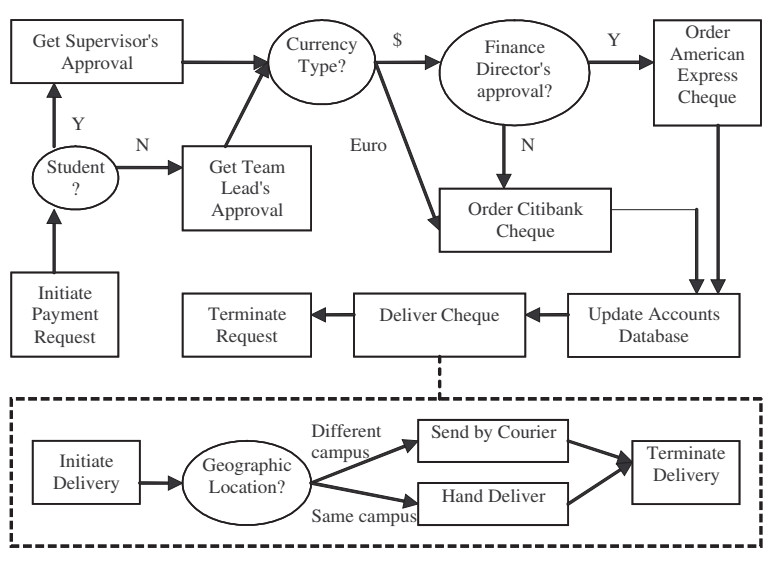

Figure 1. Travel funds request workflow.

Example 1 We consider in Fig. 1 a travel funds request service, inspired by the workflow in [12]. It involves different departments across organizations.

We model the service using the FSM $M=\left(S, s_{0}, s_{f}, \mathcal{T}\right)$ representation, as shown in Fig. 2. Notice that this FSM is a simplification of the service, since for instance the choice between the team leader or supervisor approvals is not represented. The reason is that they are both associated with an empty compensating transition, hence knowing which path was taken here is not necessary to be able to perform recovery. However, it is necessary to know which bank issued the cheque in order to able to compensate it, by a "Cancel Last American Express (Citibank) Cheque". It is also possible to handle data being written to the database. For instance, if there is no "Cancel Last Cheque" mechanism, it is possible to force the transition "Update Accounts Database" to be observable, which would lead to the exact amount of the cheque being written to the log, and recovery would manually credit the amount of money written in the log to the corresponding account.

Now, let $\mathcal{T}_{O}=\left\{e_{2}, e_{3}\right\}$ and a failure occurs while processing $e_{8}$, that is, the cheque is not issued or deliv-

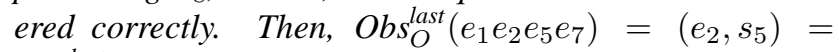
$\mathrm{Obs}_{O}^{\text {last }}\left(e_{1} e_{2} e_{4} e_{6} e_{7}\right)$. Thus, we do not know if an American Express or Citibank cheque was processed. With $\mathcal{T}_{O}^{\prime}=\left\{e_{2}, e_{6}\right\}$, we have Obs $s_{O}^{\text {last }}\left(e_{1} e_{2} e_{5} e_{7}\right)=\left(e_{2}, s_{5}\right)$ and $\operatorname{Obs}_{O}^{\text {last }}\left(e_{1} e_{2} e_{4} e_{6} e_{7}\right)=\left(e_{2} e_{6}, s_{5}\right)$, and $\mathcal{T}_{O}^{\prime}$ is an observable set of transitions. Notice that every path from $s_{0}$ to $s_{f}$ uses $\mathcal{T}_{O}^{\prime}$.

We first relate the problem of computing $M O(M)$ using our definition of observable projections with other known problems. We state now that computing the minimal observable set is equivalent to the uniconnected subgraph problem. This problem is also called the minimal marker

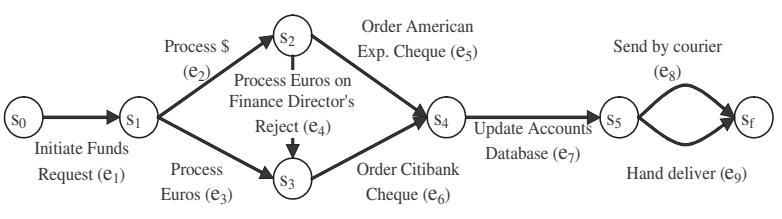

Figure 2. FSM representation of Fig. 1.

placement problem [9], in the meaning of the following proposition.

Proposition 1 Let $M$ be an FSM and $\mathcal{T}_{O}$ a subset of transitions of $M$. Denote by $M^{\prime}$ the FSM $M$ obtained by deleting all transitions belonging to $\mathcal{T}_{O}$. Then, $\mathcal{T}_{O}$ is an observable set of $M$ iff there does not exist a pair of paths $\rho_{1} \neq \rho_{2}$ of $M^{\prime}$ with $\rho_{1}$ beginning and ending at the same pair of states as $\rho_{2}$.

This problem is NP-complete [9], even with strong restrictions on the graph (acyclic, small indegree and outdegree) [5]. Moreover, this problem cannot be approximated [11] in polynomial time. It means that it is impossible to find algorithms which approximate an observable size close to the absolute minimal size (within a fairly limiting bound), for all FSMs. Anyway, one can try to identify subclasses of FSMs for which this is possible. For instance, hierarchical FSMs are a natural class of FSMs for which the problem can be solved in an efficient way [5]. However, not all FSMs used for modeling services are hierarchical. We propose here fast algorithms which give an observable set of transitions for every FSM. We will then analyze experimentally how far they are from a minimal set, and how often they are far away from the absolute minimal. Indeed, following [11], we know that there will always exist FSMs for which our polynomial time algorithms will be far away from the minimal, but the question which is of interest is how likely it is for such FSMs to occur.

\section{3 (Positively) Discriminating Algorithm}

\subsection{A Quadratic Time Algorithm}

Our first idea is to use the observation as a discriminator. When an observable transition is logged, it allows not only to know that this transition occurred (positive discrimination), but also that other implied transitions occurred. That is, the occurrence of some transitions can be deduced from the occurrence of another transition. For example, with reference to Fig. 2, if $e_{5}$ occurred, we can also infer that the transitions $e_{1}, e_{2}, e_{7}$ have occurred. The intuition is to not consider a transition as possibly observable if its occurrence 

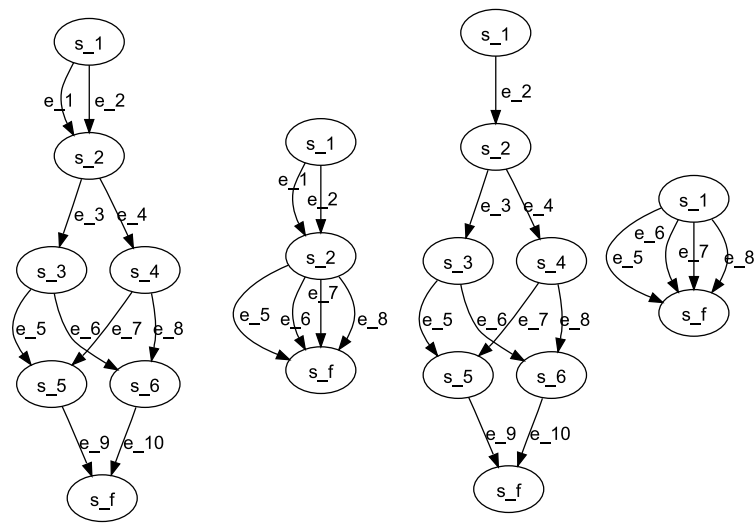

Figure 3. FSM (a), after compression (b), after deleting $e_{1}$ (c) and compressing again (d).

can be inferred from the occurrence of other transitions. We thus simply delete such transitions, using the algorithm Compress defined below.

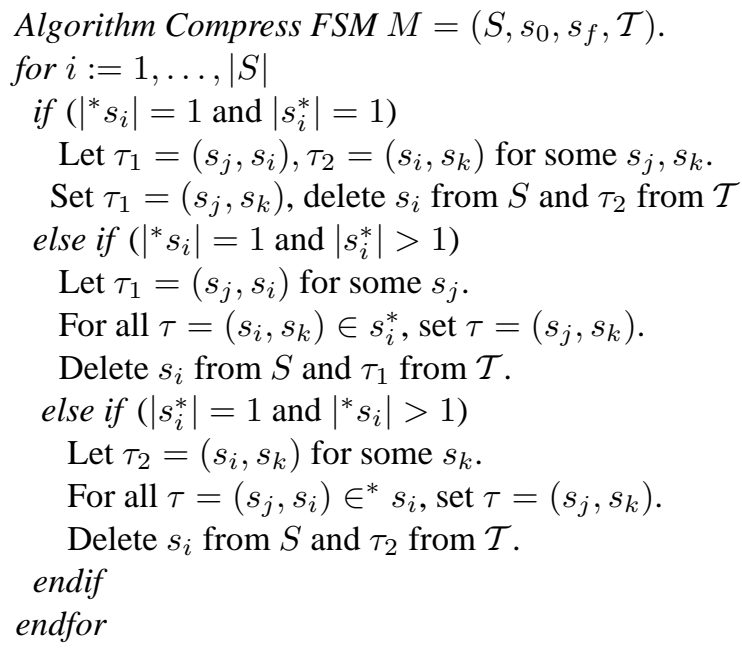

The FSM $M$ in Fig. 3(a), after compression is shown in Fig. 3(b). It is pretty clear that observing all the remaining transitions $\left\{e_{1}, e_{2}, e_{5}, e_{6}, e_{7}, e_{8}\right\}$ is an observable set of $M$, as shown in the next proposition.

Proposition 2 Given a service $M=\left(S, s_{0}, s_{f}, \mathcal{T}\right)$, the subset of transitions $\mathcal{T}^{\prime} \subseteq \mathcal{T}$ obtained on applying algorithm compress to $M$, is an observable set of transitions of $M$.

Proof. We show that for each pair of states $s_{1} \neq s_{2} \in S$, having more than one distinct path $\rho_{1} \cdots \rho_{n}$ between them, at least one transition of each of the paths is in $\mathcal{T}^{\prime}$. Without loss of generality, we consider the different cases with respect to $\rho_{1}$. Then, we have the following cases:
- $\rho_{1}$ is a straight line, that is, all intermediate states in $\rho_{1}$ have indegree and outdegree equal to 1 . Then, it is easy to see that the outgoing transition of $s_{1}$ along $\rho_{1}$ would be in $\mathcal{T}^{\prime}$.

- At least one intermediate state in $\rho_{1}$ has indegree $>1$. Let us consider the first intermediate state having indegree $>1$, say $s_{i}$. Traverse backward (towards $s_{1}$ ) from $s_{i}$ along $\rho_{1}$ till we encounter a state having outdegree $>1$, say $s_{j}$. Then, the subpath of $\rho_{1}$ from $s_{j}$ to $s_{i}$ is a straight line. And, the outgoing transition of $s_{j}$ along $\rho_{1}$ will be in $\mathcal{T}^{\prime}$.

- At least one intermediate state in $\rho_{1}$ has outdegree $>1$. Analogous to the above case.

Notice that the observable set $\mathcal{T}^{\prime}$ obtained by compression has scope for optimization, as for each pair of states $s_{1} \neq s_{2}$ of $M$ having $>1$ paths between them, the transitions in $\mathcal{T}^{\prime}$ cut all paths (as proven earlier). Recall that the existence of one path between a pair of states is not an issue with respect to observability. Thus, instead of selecting all the remaining transitions, let us just select one transition $\tau_{1}$ and declare it as observable. For instance, in Fig. 3, let $\tau_{1}=e_{1}$. We can delete it from the original FSM since it is positively discriminated (giving Fig. 3(c)). Now, imagine that some state ( $s_{2}$ in the example) had two incoming transitions before, $\tau_{1}, \tau_{2}\left(\tau_{2}=e_{2}\right.$ here $)$. After deletion of $\tau_{1}$, the state has only one incoming transition, which means that $\tau_{2}$ will get deleted by the compress algorithm (see Fig. 3(d)). Basically, it means that if we compress, select one transition at a time, delete it from the FSM, and compress again, we end up (in that case) with strictly fewer observable transitions than before ( $\tau_{2}$ is not in the observation set anymore, and we need to observe 4 transitions instead of 5). This gives rise to the following algorithm.

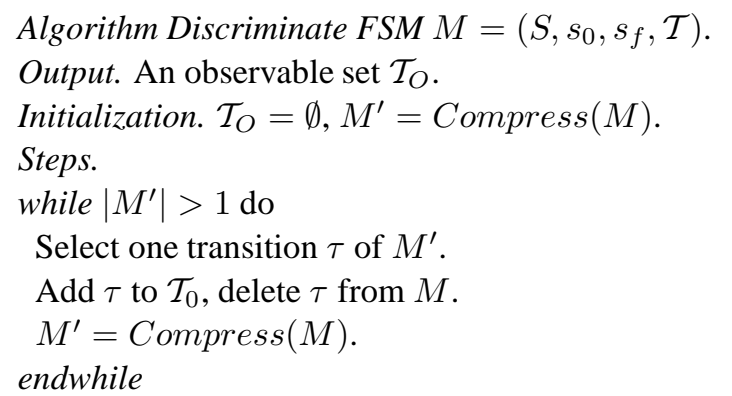

Proposition 3 For a given service $M=\left(S, s_{0}, s_{f}, \mathcal{T}\right)$, the output $\mathcal{T}_{O}$ of the discriminating algorithm is an observable set of $M$.

The problem of this algorithm is that it is in quadratic time: we need to consider every transition twice for each 
compress step, then we need to call it once for every observed transition. We will now try to characterize the selected observable set of transitions algebraically to obtain an efficient algorithm.

\subsection{Algebraic Characterization}

Let us define an extension of the classical incidence matrix of a directed acyclic graph (and of a FSM seen as a directed graph), which encodes a directed graph in a matrix. Its first rows express the directed graph, and its last rows express the observation from an observable set of transitions $\mathcal{T}_{O}$. For convenience, we index the matrix by states and transitions rather than numbers.

Definition 3 (extended incidence matrix) Let $M=\left(Q, s_{0}, s_{f}, \mathcal{T}\right)$ be an FSM and $\mathcal{T}_{O} \subseteq \mathcal{T}$ be a subset of transitions of $M$. The incidence matrix of $M$, relative to $\mathcal{T}_{O}$ is a matrix $A_{M, \mathcal{T}_{O}}$ of size $\left(|Q|+\left|\mathcal{T}_{O}\right|\right) \times|\mathcal{T}|$ defined as follows:

For every $(s, \tau) \in Q \times \mathcal{T}$ :

- $A_{M, \mathcal{T}_{O}}[s, \tau]=1$ if $\tau$ ends in $s$;

- $A_{M, \mathcal{T}_{O}}[s, \tau]=-1$ if $\tau$ starts from $s$;

- $A_{M, \mathcal{T}_{O}}[s, \tau]=0$, otherwise.

For every $\left(\tau, \tau^{\prime}\right) \in \mathcal{T}_{O} \times \mathcal{T}$ :

- $A_{M, \mathcal{T}_{O}}[\tau, \tau]=1$;

- $A_{M, \mathcal{T}_{O}}\left[\tau, \tau^{\prime}\right]=0$, if $\tau^{\prime} \neq \tau$.

The first $|Q|$ rows of this matrix correspond exactly to the classical incidence matrix. We just append to it a (almost identity) $\left|\mathcal{T}_{O}\right| \times|\mathcal{T}|$ matrix in order to obtain an extended incidence matrix. For example, the matrix corresponding to the FSM $M$ in Fig. 3(a) and $\mathcal{T}_{O}=\left\{e_{1}, e_{5}, e_{6}, e_{7}, e_{8}\right\}$, is shown in Fig. 4.

Furthermore, as we translated an FSM $M=$ $\left(Q, s_{0}, s_{f}, \mathcal{T}\right)$ into an algebraic object $A_{M, \mathcal{T}_{O}}$, we do now transform a path $\rho$ of $\mathcal{P}(M)$ into an algebraic object. Let us denote by $\chi(\rho)$ the vector in $\{0,1\}^{|\mathcal{T}|}$, such that $\chi(\rho)[\tau]=$ 1 if $\tau$ is fired in $\rho$, otherwise $\chi(\rho)[\tau]=0$. Notice that $\chi(\rho)$ characterizes any path $\rho$ of an acyclic graph (the order of transitions can be recovered unambiguously). We now define the observation $V_{M, \mathcal{T}_{O}}(X)$ associated with a vector $X$ in $\{0,1\}^{|\mathcal{T}|}$ representing a path.

Definition 4 (observation vector) The observation of $a$ vector $X$ in $\{0,1\}^{|\mathcal{T}|}$, relative to $\mathcal{T}_{O}$ is a vector $V_{M, \mathcal{T}_{O}}(X)$ of size $|Q|+\left|\mathcal{T}_{O}\right|$, such that for every $s \in Q$

- $V_{M, \mathcal{T}_{O}}(X)[s]=-1$ if $X$ starts from $s$;

- $V_{M, \mathcal{T}_{O}}(X)[s]=1$ if $X$ ends in $s$;

\begin{tabular}{c|cccccccccc} 
& $e_{1}$ & $e_{2}$ & $e_{3}$ & $e_{4}$ & $e_{5}$ & $e_{6}$ & $e_{7}$ & $e_{8}$ & $e_{9}$ & $e_{10}$ \\
\hline$s_{1}$ & -1 & -1 & 0 & 0 & 0 & 0 & 0 & 0 & 0 & 0 \\
$s_{2}$ & 1 & 1 & -1 & -1 & 0 & 0 & 0 & 0 & 0 & 0 \\
$s_{3}$ & 0 & 0 & 1 & 0 & -1 & -1 & 0 & 0 & 0 & 0 \\
$s_{4}$ & 0 & 0 & 0 & 1 & 0 & 0 & -1 & -1 & 0 & 0 \\
$s_{5}$ & 0 & 0 & 0 & 0 & 1 & 0 & 1 & 0 & -1 & 0 \\
$s_{6}$ & 0 & 0 & 0 & 0 & 0 & 1 & 0 & 1 & 0 & -1 \\
$s_{7}$ & 0 & 0 & 0 & 0 & 0 & 0 & 0 & 0 & 1 & 1 \\
$e_{1}$ & 1 & 0 & 0 & 0 & 0 & 0 & 0 & 0 & 0 & 0 \\
$e_{5}$ & 0 & 0 & 0 & 0 & 1 & 0 & 0 & 0 & 0 & 0 \\
$e_{6}$ & 0 & 0 & 0 & 0 & 0 & 1 & 0 & 0 & 0 & 0 \\
$e_{7}$ & 0 & 0 & 0 & 0 & 0 & 0 & 1 & 0 & 0 & 0 \\
$e_{8}$ & 0 & 0 & 0 & 0 & 0 & 0 & 0 & 1 & 0 & 0
\end{tabular}

Figure 4. Matrix of the FSM $M$ in Fig. 3(a) and $\mathcal{T}_{O}=\left\{e_{1}, e_{5}, e_{6}, e_{7}, e_{8}\right\}$.

- otherwise $V_{M, \mathcal{T}_{O}}(X)[i]=0$.

Moreover, for every $\tau \in \mathcal{T}_{O}$, we have:

- $V_{M, \mathcal{T}_{O}}(X)[\tau]=1$ if $X[\tau]=1$;

- otherwise $V_{M, \mathcal{T}_{O}}(X)[\tau]=0$.

Clearly, $\mathcal{T}_{O}$ is an observable set of transitions iff there does not exist $X \neq Y$ with $V_{M, \mathcal{T}_{O}}(X)=V_{M, \mathcal{T}_{O}}(Y)$. We use now the algebraic characterization.

Proposition 4 Let $X, Y \in\{0,1\}^{|\mathcal{T}|}$. Then we have:

$$
\begin{gathered}
V_{M, \mathcal{T}_{O}}(X)=V_{M, \mathcal{T}_{O}}(Y) \\
\Leftrightarrow \\
A_{M, \mathcal{T}_{O}} \cdot Y=V_{M, \mathcal{T}_{O}}(X)=A_{M, \mathcal{T}_{O}} \cdot X
\end{gathered}
$$

Using proposition 4 , we obtain that $A_{M, \mathcal{T}_{O}}$ is injective implies that $\mathcal{T}_{O}$ is an observable set of transitions. It can be shown that the discriminating algorithm gives a minimal set for which $A_{M, \mathcal{T}_{O}}$ is injective, thus giving us the algebraic characterization we were looking for (we also checked that fact experimentally).

Now, it is well known that a matrix is injective iff its kernel has dimension 0 . In our case, it is also well known [6] that the kernel of the incidence matrix $A_{M, \emptyset}$ corresponds exactly to the so-called cycle space of $M$. Moreover, the dimension of the cycle space (also known as the cyclomatic number) of a graph with $n$ vertices, $m$ edges and $K$ connected components (considering edges are unoriented) is exactly $m-n+K$. That is, it suffices to observe $m-n+K$ edges to have an injective matrix (and equivalently an observable set of transitions). That is, $M O(M) \leq m-n+K$. 


\subsection{Analysis of the Matrix Algorithm}

The matrix algorithm is clearly very fast to give an approximation of $M O(M)$, since the starting graph is connected. It suffices to count $m-n+1$ to know the number of observable transitions output by the discriminating algorithm, which is an immediate number to compute. For instance, in the example of Fig. 3, $10-7+1=4$. Knowing the transitions to log is not much harder to compute either. It suffices to run the algorithm incrementally, selecting one transition at a time. Let us now analyze which transitions are important to log. Assume we are choosing to $\log$ some transition $\tau$. Either deleting $\tau$ increases the number of connected components of $M$ or not. If it increases the number of connected components, logging it leads to the matrix algorithm on the resulting FSM giving us the transition to observe, plus $m^{\prime}-n^{\prime}+K^{\prime}$, where $m^{\prime}, n^{\prime}, K^{\prime}$ are the values for the new FSM, that is, $1+m^{\prime}-n^{\prime}+K^{\prime}=$ $1+(m-1)-n+(K+1)=m-n+K+1$. Clearly, logging this transition is not a good idea since it increases the number of transitions to $\log$ in the end. Hence, we shall never log a transition whose deletion increases the number of connected components.

On the other hand, if deleting $\tau$ keeps the number of connected components constant, then $1+m^{\prime}-n^{\prime}+K^{\prime}=$ $1+(m-1)-n+K=m-n+K$, and the transition we are choosing is useful. That is, any set of $m-n+1$ transitions whose deletion keeps the FSM connected is an observable set of transitions. To sum up, we know that we can select any transition, as long as its deletion does not disconnect connected components. Choosing with care the transition we select may optimize the result. For that, we need some heuristical metrics to tell us which transition is or is not good to observe.

Let us analyze the algorithm. Notice first that thinking in terms of positive discrimination is not always good. Indeed, in the example of Fig. 3, the compressed versions are shown in Fig. 3(b and d), leading to the observable set $\left\{e_{1}, e_{5}, e_{6}, e_{7}\right\}$. However, a minimal observation set is $\left\{e_{1}, e_{4}, e_{10}\right\}$, and observing any transition in $e_{5}, e_{6}, e_{7}, e_{8}$ leads to observing at least 4 transitions in order to get an observable set.

We call initial (final) state a state which has no incoming (outgoing) transitions. In the matrix algorithm, there can be more than one initial and/or final states. Indeed, in Fig. 3, if we delete $e_{4}$, then $s_{4}$ becomes an initial state. However, the matrix algorithm does not consider which transition is reachable from which initial state, and which final state it can reach. In particular, it is possible that $m-n+K \neq 0$, but that the FSM is observable with the current set of observable transitions (no more transitions are needed), while the matrix algorithm still asks to select some more. For example, with reference to Fig. 3, after deletion of $e_{1}, e_{4}, e_{10}, m-n+1 \neq 0$, but $\left\{e_{1}, e_{4}, e_{10}\right\}$ is already an observable set.

\section{Distinguishing Algorithm}

Based on the second observation, we design the following method to select transitions to be observed:

1. Select a transition $e$ whose deletion does not disconnect the FSM.

2. Ensure that there is an initial state $s_{0}$, a state $s$ and two paths between $\left(s_{0}, s\right)$, one using $e$ and one not using $e$ (if it is not the case, then this transition is useless for observability).

With such a method, we are sure to get at most as many transitions as observed by the matrix algorithm. Now, notice that the first condition is actually implied by the second condition. Indeed, if there exists two states $s, t$, two paths $e_{1} \cdots e_{m}, f_{1}, \cdots f_{n}$ and $e_{i} \notin\left\{f_{j} \mid j \leq n\right\}$, then it means that deleting $e_{i}$ cannot disconnect the FSM, since the undirected path $e_{i-1} \cdots\left(e_{1}=f_{1}\right) \cdots\left(f_{n}=e_{m}\right) \cdots e_{i+1}$ allows to indirectly connect $e_{i-1}{ }^{*}={ }^{*} e_{i}$ and ${ }^{*} e_{i+1}=e_{i}^{*}$. So, we can just delete the first condition. Here is the algorithm we use for testing the second condition in an efficient manner, based on Depth First Search (DFS). Basically, the algorithm breaks and returns $t$ as soon as one transition $t$ points to a state $s^{\prime}$ which has been previously explored (that is, there is another path, not using $t$ which reaches $s^{\prime}$ from an initial state $s$ from which $t$ is reachable. That is, we have two paths connecting $s$ to $s^{\prime}$, one using $t$ and one not using $t$ ). DFS keeps a stack $S$, a hash table $H$ of states which have already been explored by the search, and each transition is tagged as explored or unexplored. S.head designates the head of the stack, and t.dest the target state of a transition $t$.

Algorithm Test.

Create Hash table $H$.

for each initial state $s_{0}$ of $M$.

Initialize $H$ to empty, $S$ to $s_{0}$.

Set all the tags to unexplored.

Run DFS from $s_{0}$ :

while $S$ is nonempty do

while there is an unexplored transition $t$ from state $S$.head do

Tag $t$ as explored.

if $t$.dest $\in H$, then return $t$.

else insert $t$.dest into $H$ and push $t$.dest on $S$.

endif

endwhile

pop $S$

endwhile 
endfor

return "No more transitions to explore".

Now, notice that there can be plenty of transitions which satisfy condition 2 . We would like to have a more precise metric to choose the transition to observe in that set. Rather than optimizing the number of transitions we are positively discriminating, we want now to optimize the number of transitions we distinguish the transition from. That is, we want to optimize the number of other paths not using that transition, but connecting two states which are also connected with a path using that transition. However, computing exactly that number of paths would be really inefficient. We thus propose an efficient but slightly less accurate version: we want to maximize the number of initial or final states from or to which the second condition is true. It suffices simply to modify the previous algorithm, by keeping a counter associated with each transition, and not breaking when a transition hitting $H$ is seen, but by increasing the counter of that transition. Furthermore, we also increment the counter of the first transition which led to the insertion of that state into $H$ initially. The counter of a transition is increased at most once per initial state. We call this new algorithm Count. Obviously, an algorithm CountBack can be similarly devised, running from all final states and traversing transitions backwards. Now, our new distinguishing algorithm proceeds as follows:

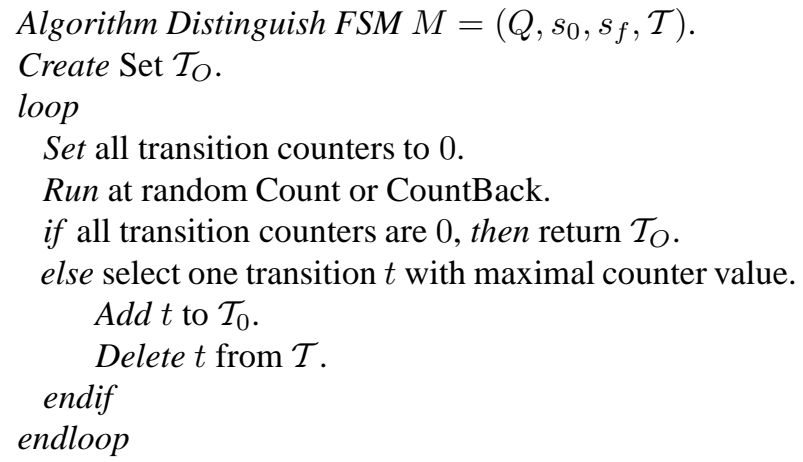

The previous claims we made allow easily to state the following.

Proposition 5 For an FSM $M=\left(Q, s_{0}, s_{f}, \mathcal{T}\right)$, the distinguishing algorithm returns an observable set $\mathcal{T}_{O}$ of transitions. Moreover, its size is always $\left|\mathcal{T}_{O}\right| \leq(|\mathcal{T}|-|Q|+1)$, which is the observable size returned by the matrix algorithm and the positively discriminating algorithm.

\section{Experimental Evaluation}

We have some theoretical clues about how our algorithms fair against each other, and how close they can approximate the absolute minimal observable size. Because our matrix and distinguishing algorithms are polynomial time, we know that there are FSMs on which they give an answer far away from the optimum [11]. The question is how far they are, and how often it happens. The second fact is that the distinguishing algorithm gives a set never bigger than the one given by the matrix algorithm. The question then is: is it better, and if yes, by how much and how often is it much better.

\begin{tabular}{|c|c|c|c|}
\hline \multirow{3}{*}{$\begin{array}{l}\text { No. of } \\
\text { edges }\end{array}$} & \multicolumn{3}{|c|}{ Observable size } \\
\hline & Absolute & Matrix & Disting. \\
\hline & 9 & 9 & 9 \\
\hline 58 & 14 & 14 & 14 \\
\hline 75 & 18 & 19 & 18 \\
\hline 103 & 20 & 24 & 20 \\
\hline 102 & 32 & 33 & 32 \\
\hline 123 & 31 & 33 & 32 \\
\hline 146 & 33 & 34 & 34 \\
\hline 146 & 42 & 42 & 42 \\
\hline 178 & 36 & 37 & 36 \\
\hline 185 & 38 & 38 & 38 \\
\hline 223 & 43 & 47 & 44 \\
\hline 221 & 53 & 57 & 55 \\
\hline 241 & 57 & 62 & 58 \\
\hline 273 & 51 & 57 & 53 \\
\hline 280 & 72 & 75 & 72 \\
\hline 294 & 74 & 76 & 75 \\
\hline 325 & 69 & 78 & 73 \\
\hline 326 & 75 & 79 & 76 \\
\hline 355 & 74 & 81 & 77 \\
\hline 345 & 88 & 91 & 88 \\
\hline 382 & 84 & 90 & 85 \\
\hline 387 & 92 & 96 & 94 \\
\hline 410 & 81 & 87 & 83 \\
\hline 445 & 89 & 97 & 90 \\
\hline 448 & 100 & 106 & 104 \\
\hline 460 & 101 & 108 & 105 \\
\hline 484 & 99 & 107 & 103 \\
\hline 489 & 104 & 111 & 107 \\
\hline 540 & 106 & 122 & 112 \\
\hline 550 & 93 & 101 & 95 \\
\hline 570 & 108 & 118 & 111 \\
\hline 605 & 108 & 124 & 111 \\
\hline 618 & 121 & 136 & 125 \\
\hline 634 & 120 & 131 & 124 \\
\hline 631 & 115 & 121 & 118 \\
\hline 657 & 131 & 141 & 134 \\
\hline 672 & 134 & 146 & 139 \\
\hline 699 & 126 & 141 & 131 \\
\hline 704 & 142 & 156 & 150 \\
\hline
\end{tabular}

\begin{tabular}{|c|c|c|}
\hline No. of & & Obs. size \\
\hline Edges & Matrix & Distinguishing \\
\hline 97 & 41 & 11 \\
\hline 127 & 55 & 13 \\
\hline 85 & 29 & 19 \\
\hline 115 & 47 & 20 \\
\hline 137 & 52 & 23 \\
\hline 126 & 43 & 27 \\
\hline 264 & 120 & 20 \\
\hline 312 & 143 & 22 \\
\hline 173 & 63 & 36 \\
\hline 201 & 77 & 52 \\
\hline 431 & 197 & 28 \\
\hline 452 & 205 & 40 \\
\hline 103 & 22 & 17 \\
\hline 200 & 72 & 39 \\
\hline 133 & 36 & 25 \\
\hline 356 & 147 & 74 \\
\hline 301 & 131 & 45 \\
\hline 114 & 27 & 19 \\
\hline 785 & 367 & 40 \\
\hline 169 & 45 & 30 \\
\hline 98 & 16 & 16 \\
\hline 101 & 17 & 17 \\
\hline 175 & 49 & 34 \\
\hline 987 & 462 & 72 \\
\hline 132 & 29 & 24 \\
\hline 490 & 203 & 113 \\
\hline 464 & 188 & 110 \\
\hline 158 & 37 & 27 \\
\hline 115 & 17 & 17 \\
\hline 620 & 268 & 140 \\
\hline 121 & 17 & 17 \\
\hline 631 & 268 & 141 \\
\hline 1013 & 468 & 90 \\
\hline 128 & 18 & 18 \\
\hline 165 & 53 & 37 \\
\hline 114 & 56 & 56 \\
\hline 214 & 58 & 36 \\
\hline 137 & 19 & 19 \\
\hline 508 & 223 & 86 \\
\hline 228 & 53 & 39 \\
\hline 747 & 315 & 178 \\
\hline 222 & 55 & 38 \\
\hline 947 & 416 & 217 \\
\hline 790 & 340 & 219 \\
\hline 161 & 23 & 23 \\
\hline 1023 & 447 & 257 \\
\hline 225 & 46 & 34 \\
\hline 997 & 431 & 230 \\
\hline 311 & 89 & 60 \\
\hline 253 & 58 & 40 \\
\hline 923 & 419 & 106 \\
\hline 321 & 90 & 63 \\
\hline 182 & 24 & 24 \\
\hline 1110 & 480 & 276 \\
\hline
\end{tabular}

\section{Figure 5. Raw data for hierarchical (left) and general (right) FSMs.}

The first question is difficult to answer accurately, since obtaining the absolute minimal observable size is intractable. One solution could be to look at small enough FSMs to get the values, but the problem is a variation of one observable transition having a big impact percentage wise in small sets, so the results would not be very meaningful. Instead, we focus on particular non-trivial FSMs, 
namely hierarchical FSMs [2]. For instance, the system in Fig. 1 is hierarchical, with 2 components. In [5], we present a polynomial time divide and conquer algorithm to compute the minimal observable size of a hierarchical FSM based on the observable sizes of its components. This allows us to compute the absolute minimal observable size of large hierarchical FSMs, as long as the components are small enough. We thus performed our two heuristics plus the absolute minimal algorithm on hierarchical FSMs, giving the results on the left part of Fig. 5, analyzing the data in the next section. Furthermore, to confirm or infirm the conclusions we draw on hierarchical FSMs, we also performed experiments for our two heuristics on general FSMs, whose results are given on the right part of Fig. 5.

\subsection{Hierarchical FSMs}

We generate hierarchical FSMs randomly, using the following method for each FSM. First, we choose a number (between one and forty) of base subcomponents in the FSM. Then, we generate each of them randomly by using the Synthetic DAG Generation Tool

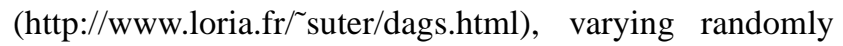
the input parameters, to get FSMs as diverse as possible. We then generate inductively a hierarchical FSM having these base components. On those FSMs, we run the algorithm from [5] to get the absolute minimal observable size, as well as the matrix and distinguishing algorithms. Fig. 6 shows the result we obtained, according to the number of transitions of the global FSM.

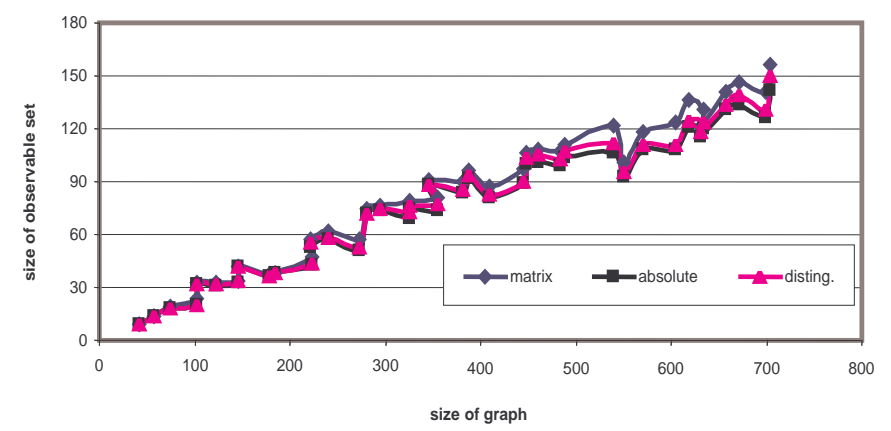

Figure 6. Observable size vs. number of transitions, over $\mathbf{4 0}$ randomly generated hierarchical FSMs.

The graph confirms that the matrix algorithm gives worse results than the distinguishing algorithm, which gives worse results than the absolute algorithm, but the difference does not seem very important.

Let us analyze more precisely the percentage difference between the absolute minimal observable size and the ob-

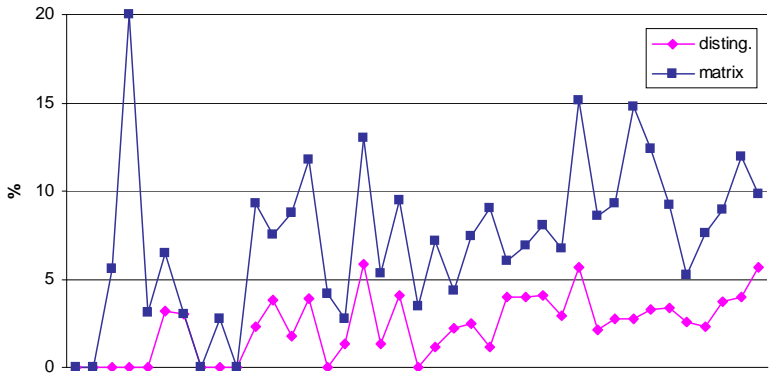

Figure 7. Deviation in percentage from the absolute minimal observable size over 40 randomly generated hierarchical FSMs.

servable sizes given by the matrix and distinguishing algorithms, on the same data (see Fig. 7). It seems that the distinguishing algorithm comes much closer to the absolute minimum, from $0 \%$ to $6 \%, 2 \%$ in mean value. The matrix algorithm is sometimes as good as the absolute minimum, sometimes much worse $(20 \%$ more transitions need to be logged), $6 \%$ in mean value, that is 3 times more than the distinguishing algorithm.

Last, we can analyze the percentage of transitions logged by the different algorithms (see Fig. 8). As mentioned in the beginning, this number is quite close for the 3 algorithms, ranging from $17 \%$ to $33 \%$. In mean values, the algorithms needs to $\log 20 \%, 21 \%$ and $22 \%$ of transitions, respectively. In terms of time taken, the matrix algorithm is instantaneous for our biggest FSM (700 transitions), the distinguishing algorithm takes 2.5 seconds to finish, and the absolute minimum takes half an hour.

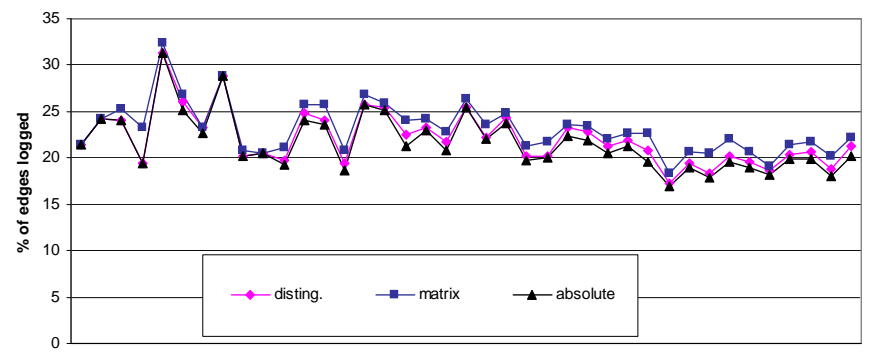

Figure 8. Percentage of edges logged by the different algorithms, over $\mathbf{4 0}$ randomly generated hierarchical FSMs. 


\subsection{General FSMs}

As mentioned previously, our first analysis is made only on particular FSMs, hence no general conclusions can be made by considering only hierarchical FSMs. We now turn to more general FSMs (on which however we cannot know the absolute minimum), to get a clue whether our first conclusions are true or not. We again use the Synthetic DAG Generation Tool, with random parameters for each size of FSM from 80 to 1200 transitions.

Fig. 9 shows the result we obtained using the matrix and distinguishing algorithms, according to the number of transitions of the FSM. The graph shows a much more chaotic picture than the one obtained on hierarchical FSMs. Furthermore, the distinguishing algorithm seems to often do much better than the matrix algorithm. Still, there are several cases (around 100 transitions) where both give the same results. Concerning time, the distinguishing algorithm takes at most 15 seconds to perform (remark that the time taken is proportional to the number of transitions logged rather than to the number of transitions in the FSM).

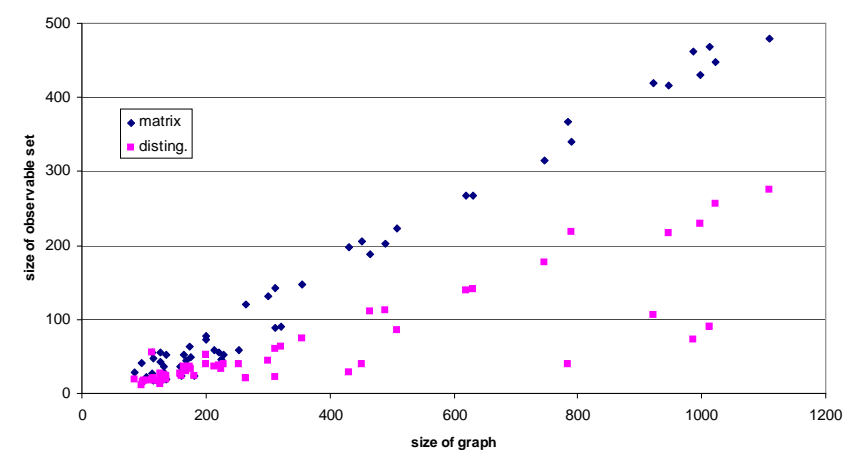

Figure 9. Observable size vs. number of transitions, over 60 randomly generated general FSMs.

Let us now analyze the percentage of transitions logged by the different algorithms (Fig. 10). Again, we see the chaosness of the picture, ranging from $15 \%$ to $50 \%$ of transitions logged by the matrix algorithm (mean value $34 \%$ ), and from $4 \%$ to $50 \%$ for the distinguishing algorithm (mean value $18 \%$ ). The comparison with results obtained on hierarchical FSMs (Fig. 8) is quite interesting. The percentage of transitions can vary from 1 to 10 , while it was from 1 to 2 in the hierarchical case. The matrix does much worse in mean value ( $34 \%$ vs $22 \%$ ), which is understandable since the FSMs are less regular and more complicated than in the hierarchical case. On the other hand, the distinguishing algorithm succeeds slightly better on this unrestricted FSMs than on hierarchical FSMs (18\% vs $21 \%)$.

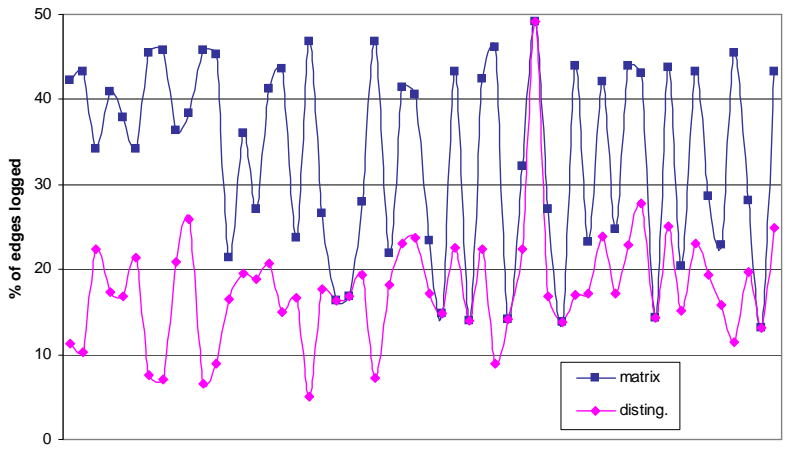

Figure 10. Percentage of edges logged by the matrix and distinguishing algorithms, over 60 randomly generated general FSMs.

Finally, we give a summing up graph in Fig. 11, where we put each random FSM we generated according to the percentage of transitions logged by the matrix algorithm and by the distinguishing algorithm, together with two broken lines labelled by $18 \%$ (vertically for the distinguishing algorithm) and 34\% (horizontally for the matrix algorithm) showing the mean values of the percentage of transitions logged. On this graph, we can draw the line (broken line labelled $100 \%$ ) on which both algorithms perform similarly. We can see that it happens several times, but mainly when the matrix algorithm already gives good results (from $12 \%$ to $18 \%$ of transitions logged, which shall be close if not equal to the optimal). Only once, the matrix is bad and so is the distinguishing algorithm (around 50\% of transitions logged). It was to be expected that such cases occur, since we know that the absolute minimal is not approximable, but luckily, it is pretty rare.

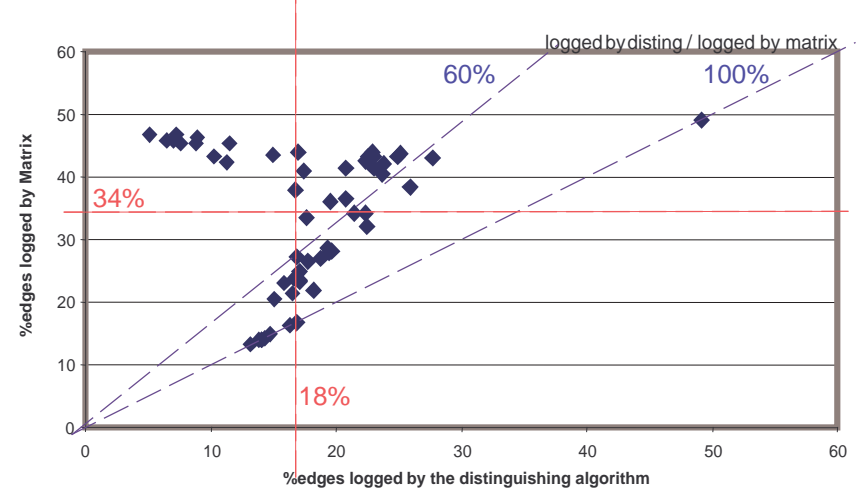

Figure 11. Percentage of edges logged by the matrix vs. distinguishing algorithm, over 60 randomly generated general FSMs. 
Overall, the distinguishing algorithm gives an observable size 0.6 times the size returned by the matrix one (we draw a broken line labelled by $60 \%$ to separate the experiments under and over that value), and almost all of its answers are within 0.7 times of the matrix algorithm. Moreover, it sometimes gives one tenth the number of transitions to log compared to the matrix algorithm (which implies that the matrix algorithm can be very inaccurate). Also, notice that only once, the distinguishing algorithm gives more than $30 \%$ of transitions to $\log$ ( $1.5 \%$ of the FSMs), while it is the case for $50 \%$ of the FSMs in the matrix algorithm.

\section{Conclusion}

We proposed two polynomial time algorithms to get an (over) approximation of the minimal number of actions to log in composite services to be able to compensate it. We modeled the services as FSMs. Our first algorithm, based on an algebraic characterization, is very fast, though it can be imprecise (in several cases, it gives at least 10 times as many transitions to log compared to the absolute minimal size). Our second algorithm based on a heuristic trying to distinguish as many conflicting transitions as possible with an observable transition, is slower but still efficient (we don't need more than 15 seconds for 1200 transitions), and usually gives much smaller observable sets. Still, in one case, it seems to give inaccurate results. There are probably some more heuristics to apply to get a more accurate algorithm. Nevertheless, in mean value, it seems that the distinguishing algorithm gives results close to the absolute minimum ( $18 \%$ of transitions, while we get $20 \%$ for the absolute minimal, looking at hierarchical FSMs), so efforts to optimize it further would probably not be worth it but for very few pathological cases, and would slow down the algorithm.

\section{References}

[1] G. Alonso, F. Casati, and H. Kuno. Web Services: Concepts, Architecture and Applications. Springer, ISBN: 3540440089, 2004.

[2] R. Alur and M. Yannakakis. Model checking of hierarchical state machines. ACM Transactions on Programming Languages and Systems (TOPLAS), 23(3), pages 1-31, 2001.

[3] P. A. Bernstein, V. Hadzilacos, and N. Goodman. Concurrency Control and Recovery in Database Systems. AddisonWesley, ISBN: 0201107155, 1987.

[4] D. Biswas. Compensation in the world of web services composition. In proceedings of the International Workshop on Semantic Web Services and Web Process Composition (SWSWPC), LNCS 3387, pages 69-80, 2004.

[5] D. Biswas and B. Genest. Minimal observability for transactional hierarchical services. In proceedings of the 20th International Conference on Software Engineering and Knowledge Engineering (SEKE), pages 531-536, 2008.
[6] C. Godsil and G. Royle. Algebraic Graph Theory. Springer, ISBN: 0387952209, 2001.

[7] R. Kumar and V. K. Garg. Modeling and Control of Logical Discrete Event Systems. Springer, ISBN: 0792395387, 1994.

[8] F. Lin and W. M. Wonham. On observability of discreteevent systems. Information Sciences, 44(3), pages 173-198, 1988.

[9] S. Maheshwari. Traversal marker placement problems are np-complete. Boulder University Research Report CU-CS092-76, 1976.

[10] C. M. Özveren and A. S. Wilsky. Observability of discrete event dynamical systems. IEEE Transactions on Automatic Control, 35(7), pages 797-806, 1990.

[11] K. Rohloff, S. Khuller, and G. Kortsarz. Approximating the minimal sensor selection for supervisory control. Discrete Event Dynamic Systems, 16(1), pages 143-170, 2006.

[12] W. Sadiq and M. E. Orlowska. Analyzing process models using graph reduction techniques. Information Systems, 25(2), pages 117-134, 2000.

[13] M. Sampath, R. Sengupta, S. Lafortune, K. Sinaamohideen, and D. Teneketzis. Diagnosability of discrete event systems. IEEE Transactions on Automatic Control, 40(9), pages 1555-1575, 1995.

[14] A. Wombacher, P. Fankhauser, and E. Neuhold. Transforming bpel into annotated deterministic finite state automata for service discovery. In proceedings of the IEEE International Conference on Web Services (ICWS), pages 316-323, 2004.

[15] T.-S. Yoo and S. Lafortune. Np-completeness of sensor selection problems arising in partially-observed discrete-event systems. IEEE Transactions on Automatic Control, 47(9), pages 1495-1499, 2002. 